\title{
Comparison of the Acute Effects of Right Ventricular Apical Pacing and Biventricular Pacing in Patients with Heart Failure
}

\author{
Hideaki Kanzaki ${ }^{1}$, Kazuhiro Satomi ${ }^{1}$, Takashi Noda ${ }^{1}$, Wataru Shimizu ${ }^{1}$, Shiro Kamakura ${ }^{1}$, \\ Yasushi Kitaura $^{2}$, Nobukazu Ishizaka ${ }^{2}$ and Masafumi Kitakaze ${ }^{1}$
}

\begin{abstract}
Objective Upgrading to biventricular (BiV) pacing benefits heart failure patients with right ventricular (RV) apical pacing. However, the impact of switching from RV apical pacing to BiV pacing on the left ventricular (LV) function accompanied by changes in the QRS duration remains unknown. We aimed to investigate the effects of BiV pacing in heart failure patients under RV apical pacing.

Methods In 37 patients with heart failure (LV ejection fraction: 22 $\pm 9 \%$ ), the maximum rate of LV pressure

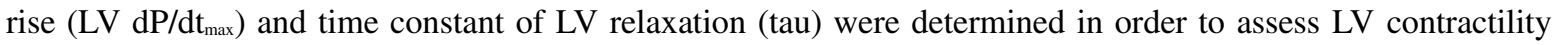
and diastolic relaxation, respectively, under RV apical pacing and BiV pacing. Switching from RV pacing to BiV pacing, the QRS duration was shortened from $209 \pm 42$ to $162 \pm 28 \mathrm{~ms}(\mathrm{p}<0.001)$ and the $\mathrm{LV} \mathrm{dP} / \mathrm{dt}_{\max }$ values were increased in all patients $(+18.4 \pm 11.3 \%, \mathrm{p}<0.001)$, whereas the $\mathrm{LV}$ tau values varied $(-1.5 \pm 13.0 \%$, $\mathrm{p}=0.723)$. Shortening of the QRS duration correlated with the increase in $\mathrm{LV} d P / \mathrm{dt}_{\max }(\mathrm{r}=-0.689, \mathrm{p}<0.001)$; however, it was not closely associated with the changes in LV tau.

Conclusion Switching from RV apical pacing to BiV pacing improves the LV contractile function in proportion to the degree of QRS shortening. BiV pacing is recommended in patients with systolic heart failure and a prolonged RV-paced QRS duration.
\end{abstract}

Key words: cardiac resynchronization therapy, heart failure, QRS duration, RV pacing

(Intern Med 54: 1329-1335, 2015)

(DOI: 10.2169/internalmedicine.54.3081)

\section{Introduction}

Cardiac resynchronization therapy (CRT) via biventricular (BiV) pacing reportedly improves the cardiac function, symptoms and prognosis in heart failure patients with a prolonged QRS duration. These benefits are primarily attributable to restoration of the asynchronous and wasteful ventricular motion induced by intraventricular conduction delays. Indeed, the maximum rate of left ventricular (LV) pressure rise $\left(\mathrm{LV} \mathrm{dP} / \mathrm{dt}_{\max }\right)$ and stroke volume immediately increase after BiV pacing (1). In addition, several studies have shown that chronic right ventricular (RV) apical pacing worsens the prognosis in patients with heart failure $(2,3)$.
RV apical pacing induces a conduction delay no less than that induced by left bundle branch block (LBBB) (4). Moreover, the delay is enhanced by concomitant conduction disease (4). RV pacing-induced dyssynchrony is a determinant of LV remodeling (5), and upgrading to CRT benefits patients with systolic heart failure and RV apical pacing (6-9).

The spread of electrical activity through the ventricular myocardium produces the QRS complex on electrocardiograms (ECGs), and a prolonged QRS duration of $\geq 120 \mathrm{~ms}$ represents a delay in ventricular activation. Auricchio et al. (10) reported that the response to CRT is markedly superior in patients with a QRS duration of $>150 \mathrm{~ms}$ than in those with a shorter QRS duration. In addition, the RethinQ study demonstrated no functional benefits from CRT among

${ }^{1}$ Department of Cardiovascular Medicine, National Cerebral and Cardiovascular Center, Japan and ${ }^{2}$ Department of Cardiology, Osaka Medical College, Japan

Received for publication April 12, 2014; Accepted for publication October 16, 2014

Correspondence to Dr. Hideaki Kanzaki, kanzakih@ncvc.go.jp 
Table 1. Baseline Parameters.

\begin{tabular}{lccc}
\hline Characteristics & $\begin{array}{c}\text { Intrinsic rhythm } \\
(\mathrm{n}=23)\end{array}$ & $\begin{array}{c}\text { Chronic RV pacing } \\
(\mathrm{n}=14)\end{array}$ & $\mathrm{p}$ \\
\hline Age, years & $56 \pm 15$ & $56 \pm 16$ & $\mathrm{~ns}$ \\
Male, $\mathrm{n}$ (\%) & $16(70)$ & $8(57)$ & $\mathrm{ns}$ \\
BSA, m ${ }^{2}$ & $1.58 \pm 0.15$ & $1.54 \pm 0.20$ & $\mathrm{~ns}$ \\
NYHA functional class, $\mathrm{n}(\%)$ & & & $\mathrm{ns}$ \\
$\quad$ II & $4(17)$ & $0(0)$ & \\
III & $18(78)$ & $12(86)$ & $\mathrm{ns}$ \\
IV & $1(4)$ & $2(14)$ & $\mathrm{ns}$ \\
LVEF, \% & $23 \pm 10$ & $21 \pm 6$ & $\mathrm{~ns}$ \\
LVDd, mm & $71 \pm 14$ & $70 \pm 11$ & $<0.001$ \\
LVDs, mm & $63 \pm 14$ & $62 \pm 11$ & $\mathrm{~ns}$ \\
Baseline QRS duration, ms & $145 \pm 39$ & $237 \pm 40$ & 0.078 \\
RV-paced QRS duration, ms & $192 \pm 34$ & $237 \pm 40$ & \\
Atrial fibrillation, $\mathrm{n}(\%)$ & $4(17)$ & $6(43)$ & \\
Etiology of heart failure, $\mathrm{n}(\%)$ & $18(78)$ & $6(43)$ & \\
Idiopathic DCM & $2(9)$ & $4(29)$ & $\mathrm{ns}$ \\
End-stage HCM & $1(4)$ & $3(21)$ & $\mathrm{ns}$ \\
Cardiac sarcoidosis & $2(9)$ & $1(7)$ & \\
$\quad$ OMI & & & \\
Medications, $\mathrm{n}(\%)$ & $20(87)$ & $11(79)$ & \\
$\quad$ Beta-blockers & $17(74)$ & & \\
ACEi / ARB & & &
\end{tabular}

Data are expressed as mean \pm SD or as number (percentage).

RV: right ventricular, BSA: body surface area, NYHA: New York Heart Association, LV: left ventricular, EF: ejection fraction, Dd: end-diastolic diameter, Ds: end-systolic diameter, DCM: dilated cardiomyopathy, HCM: hypertrophic cardiomyopathy, OMI: old myocardial infarction, ACEi: angiotensin-converting enzyme inhibitors, ARB: angiotensin II receptor blockers

patients with a QRS duration of $<120 \mathrm{~ms}$ (11). A QRS duration of $\geq 120 \mathrm{~ms}$ is the main selection criterion for CRT, Nevertheless, recent guidelines have changed the recommended QRS threshold for CRT to $\geq 150 \mathrm{~ms}(12,13)$. In other words, the QRS duration continues to be a crucial criterion in clinical routines. However, with respect to switching from $\mathrm{RV}$ apical pacing to $\mathrm{BiV}$ pacing, the impact on the LV function and accompanying changes in the QRS duration remain unknown.

The $\mathrm{LV} \mathrm{dP/dt} \mathrm{t}_{\max }$ is a common and sensitive indicator of acute changes in the LV contractile function and is dependent on the heart rate and LV preload (14). The time constant of $\mathrm{LV}$ relaxation (tau) is an accurate indicator of $\mathrm{LV}$ diastolic relaxation, independent of the loading conditions (15). Impaired LV relaxation is reflected by a prolonged tau. These parameters have been established as indices of the LV function, although their measurement requires cardiac catheterization using a high-fidelity, micromanometer-tipped catheter. Increased stiffness is also associated with LV diastolic filling abnormalities; however, it is unlikely that $\mathrm{BiV}$ pacing influences the degree of myocardial stiffness immediately. Accordingly, we aimed to investigate the effects of $\mathrm{BiV}$ pacing in patients with heart failure and RV apical pacing using $\mathrm{LV} \mathrm{dP} / \mathrm{dt}_{\max }$ and tau values derived from catheters.

\section{Materials and Methods}

\section{Study population}

We identified 50 patients who provided informed consent and underwent invasive temporary pacing studies to assess their eligibility as CRT candidates between April 2003 and December 2007. Thirteen patients were excluded from the analysis for the following reasons: high-fidelity LV pressure traces were not recorded using a micromanometer-tipped catheter, the LV pacing lead could not be positioned in the posterolateral or lateral branch of the coronary sinus or the catheter could not be inserted into the left ventricle due to aortic stenosis or the presence of a mechanical aortic valve. Consequently, we enrolled 37 patients in our study. Thirty patients (81\%) had heart failure of New York Heart Association (NYHA) functional class III, four $(11 \%)$ of class II and three $(8 \%)$ of class IV despite optimal medical therapy. The mean LV ejection fraction (LVEF) was $22 \pm 9 \%$ and the mean QRS duration was $179 \pm 60$ ms. Ten patients (27\%) had chronic atrial fibrillation. The etiology of heart failure was idiopathic dilated cardiomyopathy in 24 patients $(65 \%)$, endstage hypertrophic cardiomyopathy in six patients (17\%), cardiac sarcoidosis in four patients $(11 \%)$ and previous myocardial infarction in three patients $(8 \%)$. Fourteen patients (38\%) had chronic RV apical pacing. Table 1 shows the differences in the baseline characteristics between the patients with an intrinsic rhythm and those with chronic RV pacing. CRT devices were subsequently implanted in 27 patients, including de novo CRT in 17 cases and upgrade procedures in 10 cases. The present study was approved by the institutional review board and conducted in accordance with the ethical principles of the Declaration of Helsinki. 


\section{Test pacing study procedure}

An 8-Fr guide catheter (IS Catheter, St. Jude Medical, St. Paul, USA) or 6-Fr catheter (Goodtec CS Catheter, Goodman Co. Ltd., Nagoya, Japan) were introduced into the coronary sinus under guide-wire assistance. A 6-Fr catheter with a 20-mm occlusion balloon at the tip (Selecon MP Catheter II, Terumo Clinical Supply Co. Ltd., Gifu, Japan) was then advanced or replaced by changing the guide-wire in the proximal section of the coronary sinus. After injecting contrast medium to identify the branches of the coronary sinus, a 2.5-Fr, 16-pole pacing catheter (PATHFINDER ${ }^{\circledR}$, Cardima, Fremont, USA) was advanced into the target vein (i.e., the posterolateral or lateral branch of the coronary sinus) and positioned at the level of the mid-ventricle. A 5-Fr micromanometer-tipped pigtail catheter (Millar Mikro-Tip ${ }^{\circledR}$, Millar Instruments, Houston, USA) was inserted into the left ventricle, and a 7-Fr Swan-Ganz catheter (Edwards Lifesciences, Irvine, USA) was placed in the pulmonary artery for right-sided heart catheterization. A 6-Fr steerable four-pole pacing catheter (Boston Scientific/EP Technologies, San Jose, USA) was placed at the RV apex in patients with no preexisting leads, and a 5-Fr, four-pole pacing catheter $\left(\right.$ Bard $^{\circledR}$ Viking $^{\mathrm{TM}}$ Soft Tip, Bard Electrophysiology, Lowell, USA) was placed at the right atrial appendage in patients in sinus rhythm with no atrial leads.

The LV pressure and right heart catheterization variables were recorded when the patient was in a steady state three minutes after pacing. The cardiac output was measured according to the Fick principle based on the assumed oxygen consumption and indexed to the body surface area (cardiac index). In principle, all hemodynamic parameters were recorded at an atrial-paced heart rate of approximately 70 beats/min (bpm) with a sensed atrioventricular (AV) delay of $120 \mathrm{~ms}$, paced AV delay of $150 \mathrm{~ms}$ and interventricular (VV) delay of $0 \mathrm{~ms}$. In patients with atrial fibrillation, the pacing rate was set to $70 \mathrm{bpm}$ and the $\mathrm{VV}$ delay was set to $0 \mathrm{~ms}$. The pacing rate was adjusted at a rate just above the intrinsic rate for ventricular pacing, if required. The QRS duration was manually measured with electronic calipers from the beginning to the end of the QRS complex with simultaneous 12-lead ECG signals (maximum QRS) acquired on a computer-assisted pacing modulation system (EPWorkMate $^{\mathrm{TM}}$, EP Medsystems, West Berlin, USA).

The LV pressure signals were digitally stored to compute the $\mathrm{LV} \mathrm{dP} / \mathrm{dt}_{\max }$ and tau values using a polygraph system (RMC-2000, Nihon Kohden, Tokyo, Japan). Both the LV $\mathrm{dP} / \mathrm{dt}_{\max }$ and tau values were determined using the average value obtained over 8 seconds. The LV tau value was calculated using a monoexponential pressure decay model with the Weiss formula (15).

\section{Statistical analysis}

The data are expressed as the mean \pm standard deviation or the count (percentage). Student's unpaired and paired $t$ tests were used to compare two groups of independent and matched patients, respectively. Fisher's exact test was performed for the analysis of group differences. Linear regression was used to assess the relationships between the QRS duration and hemodynamic parameters. All statistical tests were performed using the Stata 10.1 software program (StataCorp LP, College Station, USA). A p value of $<0.05$ was considered to be statistically significant.

\section{Results}

\section{Hemodynamic effects of switching to BiV pacing}

As a result of switching from $\mathrm{RV}$ apical pacing to BiV

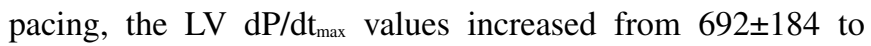
$810 \pm 184 \mathrm{mmHg} / \mathrm{s}(+18.4 \pm 11.3 \%, \mathrm{p}<0.001 ;$ Fig. 1$)$. All 37 patients showed an increase in $\mathrm{LV} \mathrm{dP} / \mathrm{dt}_{\max }, 28(76 \%)$ of whom exhibited a $>10 \%$ increase. The LV systolic pressure increased from $91 \pm 18$ to $95 \pm 18 \mathrm{mmHg}$ ( $\mathrm{p}<0.001$ ), and the $\mathrm{LV}$ end-diastolic pressure changed from $15 \pm 8$ to $13 \pm 7$ $\mathrm{mmHg}(\mathrm{p}=0.651)$. The heart rate was $75 \pm 10 \mathrm{bpm}$ and $74 \pm 10$ bpm, respectively $(-0.0 \pm 3.6 \%, \mathrm{p}=0.953)$. Finally, the cardiac index increased from $1.96 \pm 0.42$ to $2.13 \pm 0.46 \mathrm{~L} / \mathrm{min} / \mathrm{m}^{2}$ (+ $9.1 \pm 11.0 \%, \mathrm{p}<0.001$ ), although five subjects displayed no increases in the cardiac index.

In contrast, the changes in the $\mathrm{LV}$ tau values varied among the 37 patients $(-1.5 \pm 13.0 \%$, from $65.0 \pm 17.5$ to $63.1 \pm 15.0 \mathrm{~ms}, \mathrm{p}=0.723$ ), as shown in Fig. 1. Twenty-two subjects (59\%) showed an improvement in LV tau; conversely, 15 (41\%) showed deterioration or no changes. The differences in baseline characteristic between the patients with and without improvements in LV tau are summarized in Table 2, with no significant differences between the groups. Among the patients with RV apical pacing, when switching to $\mathrm{BiV}$ pacing, the resultant changes in $\mathrm{LV} \mathrm{dP} /$ $\mathrm{dt}_{\max }$ did not demonstrate a close relationship to the changes in LV tau (Fig. 2).

\section{Effects of BiV pacing on the QRS duration}

As a result of switching from $\mathrm{RV}$ pacing to BiV pacing, the QRS duration decreased from $209 \pm 42$ to $162 \pm 28 \mathrm{~ms}$ ( $\mathrm{p}<$ $0.001)$. The degree of QRS shortening correlated with the increases in $\mathrm{LV} \mathrm{dP} / \mathrm{dt}_{\max }(\mathrm{r}=-0.689, \mathrm{p}<0.001$; Fig. 3) and cardiac index $(\mathrm{r}=-0.374, \mathrm{p}=0.023)$, but not the changes in $\mathrm{LV}$ tau ( $\mathrm{r}=0.071, \mathrm{p}=0.676)$. In addition, the RV-paced QRS duration showed a very modest yet significant correlation with the increase in $\mathrm{LV} \mathrm{dP} / \mathrm{dt}_{\max }(\mathrm{r}=0.539, \mathrm{p}<0.001$; Fig. 3), but not the changes in $\mathrm{LV}$ tau $(\mathrm{p}=0.441)$. Of the 29 patients with a RV-paced QRS duration of $\geq 180 \mathrm{~ms}, 25(86 \%)$ showed a $>10 \%$ improvement in $\mathrm{LV} \mathrm{dP} / \mathrm{dt}_{\max }$. Of the $22 \mathrm{pa}-$ tients with a RV-paced QRS duration of $\geq 200 \mathrm{~ms}, 17$ (77\%) showed a $>15 \%$ improvement in $\mathrm{LV} \mathrm{dP} / \mathrm{dt}_{\max }$.

The RV-paced QRS duration was longer in the 14 patients with chronic $\mathrm{RV}$ pacing than in the 23 patients with temporary RV pacing $(237 \pm 40 \mathrm{~ms}$ vs. $192 \pm 34 \mathrm{~ms}, \mathrm{p}<0.001)$, and the improvement in LV contractility was greater in the patients with chronic RV pacing than in those with temporary 

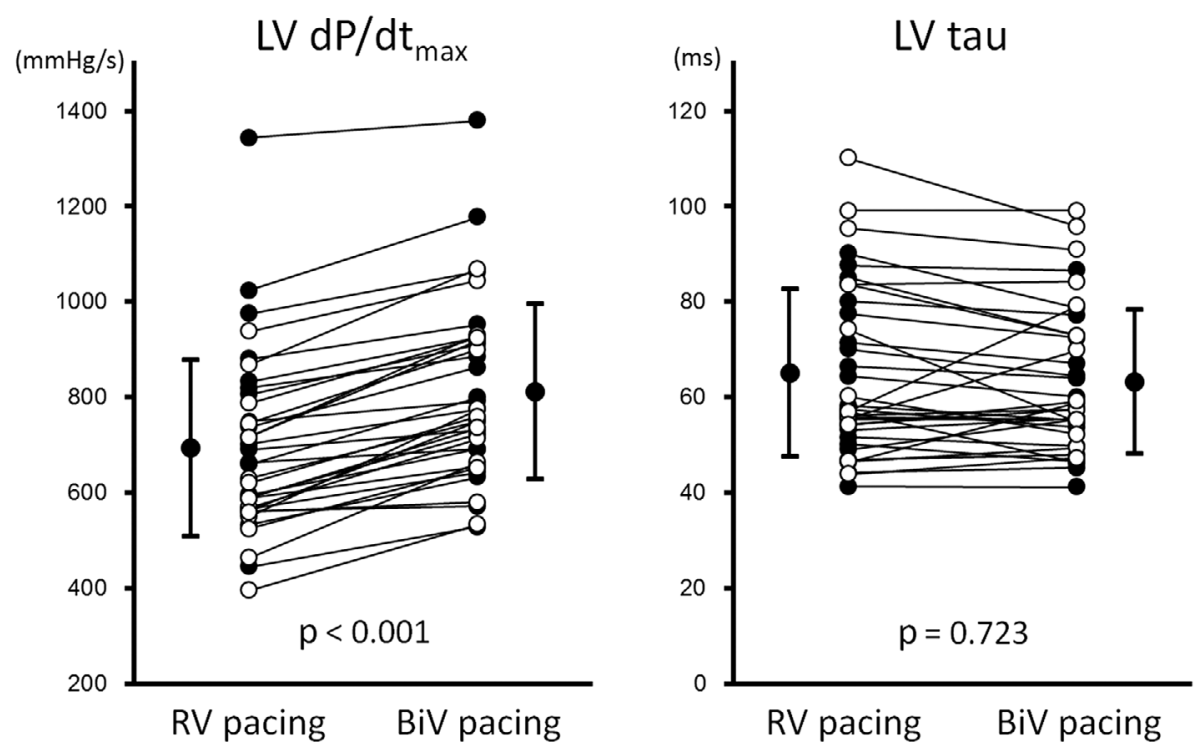

Figure 1. Changes in left ventricular $\mathrm{dP} / \mathrm{dt}_{\max }$ and tau in switching from right ventricular apical pacing to biventricular pacing. When switching from right ventricular (RV) apical pacing to biventricular pacing, left ventricular $(\mathrm{LV}) \mathrm{dP} / \mathrm{dt}_{\max }$ increases in all patients (Left); however, the change in LV tau varies between subjects (Right). ( $\bigcirc$ ), patients with chronic RV pacing; $(O)$, patients with intrinsic conduction. Abbreviations: $d P / d_{\text {max }}$ : the maximum rate of $L V$ pressure rise, tau: the time constant for isovolumic relaxation, RV: right ventricular, LV: left ventricular

Table 2. Baseline Parameters in Patients with and without Improvement in LV Diastolic Relaxation.

\begin{tabular}{|c|c|c|c|}
\hline Characteristics & $\begin{array}{c}\text { Improvement } \\
(\mathrm{n}=22)\end{array}$ & $\begin{array}{l}\text { Non-improvement } \\
\qquad(\mathrm{n}=15)\end{array}$ & $\mathrm{p}$ \\
\hline Age, years & $57 \pm 16$ & $55 \pm 15$ & ns \\
\hline Male, $\mathrm{n}(\%)$ & $14(64)$ & $10(67)$ & ns \\
\hline $\mathrm{BSA}, \mathrm{m}^{2}$ & $1.56 \pm 0.15$ & $1.57 \pm 0.20$ & ns \\
\hline NYHA functional class, n (\%) & & & ns \\
\hline II & $2(9)$ & $2(13)$ & \\
\hline III & $18(82)$ & $12(80)$ & \\
\hline IV & $2(9)$ & $1(7)$ & \\
\hline LVEF, \% & $22 \pm 9$ & $22 \pm 9$ & ns \\
\hline LVDd, mm & $74 \pm 14$ & $65 \pm 11$ & ns \\
\hline LVDs, mm & $65 \pm 14$ & $58 \pm 11$ & ns \\
\hline Baseline QRS duration, ms & $175 \pm 54$ & $187 \pm 69$ & ns \\
\hline RV-paced QRS duration, ms & $208 \pm 34$ & $210 \pm 53$ & ns \\
\hline Chronic RV pacing, n (\%) & $6(27)$ & $8(53)$ & ns \\
\hline Atrial fibrillation, $\mathrm{n}(\%)$ & $7(32)$ & $3(20)$ & ns \\
\hline Etiology of heart failure, $\mathrm{n}(\%)$ & & & 0.100 \\
\hline Idiopathic DCM & $17(77)$ & $7(47)$ & \\
\hline End-stage HCM & $3(14)$ & $3(20)$ & \\
\hline Cardiac sarcoidosis & $2(9)$ & $2(13)$ & \\
\hline OMI & $0(0)$ & $3(20)$ & \\
\hline \multicolumn{4}{|l|}{ Medications, n (\%) } \\
\hline Beta-blockers & $18(82)$ & $13(87)$ & ns \\
\hline $\mathrm{ACEi} / \mathrm{ARB}$ & $17(77)$ & $10(67)$ & ns \\
\hline
\end{tabular}

Data are expressed as mean \pm SD or as number (percentage).

RV: right ventricular, BSA: body surface area, NYHA: New York Heart Association, LV: left ventricular, EF : ejection fraction, Dd: end-diastolic diameter, Ds: end-systolic diameter, DCM: dilated cardiomyopathy, HCM: hypertrophic cardiomyopathy, OMI: old myocardial infarction, ACEi: angiotensin-converting enzyme inhibitors, ARB: angiotensin II receptor blockers

RV pacing $(24.4 \pm 10.6 \%$ vs. $14.8 \pm 10.3 \%, \mathrm{p}=0.011)$. In contrast, there were no significant differences in patient characteristics between the subjects with temporary RV pacing and those with chronic pacing, except for the baseline QRS duration and RV-paced QRS duration (Table 1). 


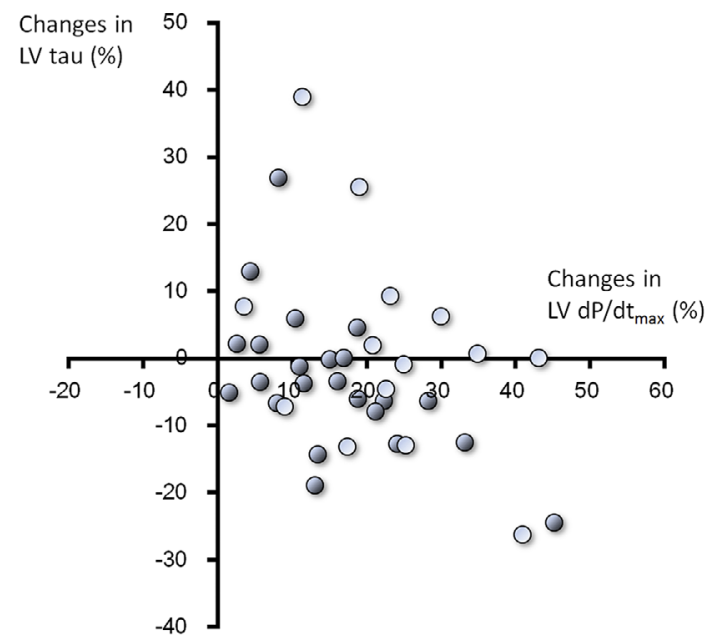

Figure 2. Distribution of changes in left ventricular $\mathbf{d P} / \mathbf{d t}_{\max }$ and tau in switching from right ventricular apical pacing to biventricular pacing. Changes in left ventricular $\mathrm{dP} / \mathrm{dt}_{\max }$ and tau are not closely associated with switching from right ventricular (RV) apical pacing to biventricular pacing. $(\bigcirc)$, patients with chronic $R V$ pacing; $(O)$, patients with intrinsic conduction. Abbreviations: $d P / d_{\text {max }}$ : the maximum rate of $L V$ pressure rise, tau: the time constant for isovolumic relaxation, RV: right ventricular

\section{Discussion}

When switching from RV apical pacing to BiV pacing, the LV contractile function immediately improved in all patients, whereas the changes in LV diastolic relaxation varied between the subjects. In the present study, we found that shortening of the QRS duration accompanied by switching from RV apical pacing to BiV pacing correlated with the improvement in the LV contractile function. However, the changes in LV diastolic relaxation were not closely associated with either the changes in the LV contractile function or QRS duration.

Whether shortening of the QRS duration following BiV pacing is associated with the benefits of CRT remains controversial. Kass et al. (1) reported that the intrinsic QRS duration at baseline positively correlates with the \%change in $\mathrm{LV} \mathrm{dP} / \mathrm{dt}_{\max }$ induced by pacing, although the pacing efficacy is not associated with QRS shortening. Meanwhile, Kashani et al. (16) reviewed numerous studies and concluded that the baseline QRS duration and QRS narrowing induced by CRT do not correlate with hemodynamic or clinical improvements. In contrast, Lecoq et al. (17) reported that the amount of QRS shortening associated with BiV pacing is an independent predictor of the response to CRT. According to our results, LV contractility improved by switching from RV pacing to BiV pacing in proportion to the degree of QRS narrowing. In addition, the beneficial effects were more remarkable for a longer RV-paced QRS duration. There is a strong association between a prolonged RV-paced QRS duration and the risk of heart failure events (3). The QRS du- ration reflects total ventricular depolarization; in particular, the RV-paced QRS duration well approximates the LV activation time (4). If $\mathrm{LV}$ or $\mathrm{BiV}$ pacing serves as an electrical bypass for conduction delay, it is not surprising that QRS narrowing is linked to an increase in LV contractile efficiency. This observation is supported by the findings of a recent study reporting that QRS narrowing predicted reverse remodeling in 112 patients with chronic RV pacing upgraded to CRT (18).

Our subjects consisted of 14 patients with chronic RV pacing and 23 patients with temporary RV pacing. The RVpaced QRS duration is longer in patients with chronic RV pacing than those with temporary RV pacing. Although the reason for this phenomenon was not clarified in this study, the RV paced-QRS duration is reported to be increased in the presence of LV dysfunction and underlying conduction system disease (4). Notably, patients who require RV pacing may also exhibit disturbances in the intraventricular conduction system. Furthermore, conduction disorders may be progressive under long-term RV pacing. However, interestingly, the improvement in LV contractility noted in the present study was greater in the patients with chronic RV pacing than in those with temporary RV pacing. Moreover, the response to BiV pacing showed a similar tendency between the patients with chronic and temporary RV pacing (Fig. 3). This finding does not necessarily contradict reports showing that the response to CRT upgrading is independent of the duration of prior RV pacing $(9,19)$.

On the other hand, the changes in LV diastolic relaxation were independent of those in the LV contractile function (Fig. 2). Among our subjects, the LV tau values did not improve in $41 \%$ of the patients. The reason for this observation remains unclear. However, a possible explanation is that, depending on the pacing site, LV pacing may disturb LV torsion, which stores potential energy during LV systole and aids in diastolic untwisting, thus impairing LV diastolic relaxation. At a minimum, changes in LV relaxation are not likely to be associated with changes in the QRS duration representing the ventricular depolarization period. In this study, no improvements in the cardiac index were detected in some subjects, despite the increase in $\mathrm{LV} \mathrm{dP} / \mathrm{dt}_{\max }$. This finding is likely due to insufficient LV preload, although it may also be partially associated with deterioration of $\mathrm{LV}$ diastolic relaxation.

Donahue et al. (20) demonstrated that, among 47 patients with an LVEF of $\leq 35 \%$, a QRS duration of $<120 \mathrm{~ms}$ and dyssynchrony, as evidenced on tissue Doppler imaging, the \%improvement in $\mathrm{LV} \mathrm{dP} / \mathrm{dt}_{\max }$ with CRT was $2 \%$ on average. Furthermore, Ruschitzka et al. (21) showed that, in 809 patients with an LVEF of $\leq 35 \%$ and QRS duration of $<130$ $\mathrm{ms}$, even if dyssynchrony was documented, CRT did not reduce the rate of death or hospitalization due to heart failure, but rather increased mortality. Our results are consistent with these reports. Therefore, in patients with a narrow QRS complex in whom obtaining further reductions in the QRS duration is difficult, adequate hemodynamic improvements 

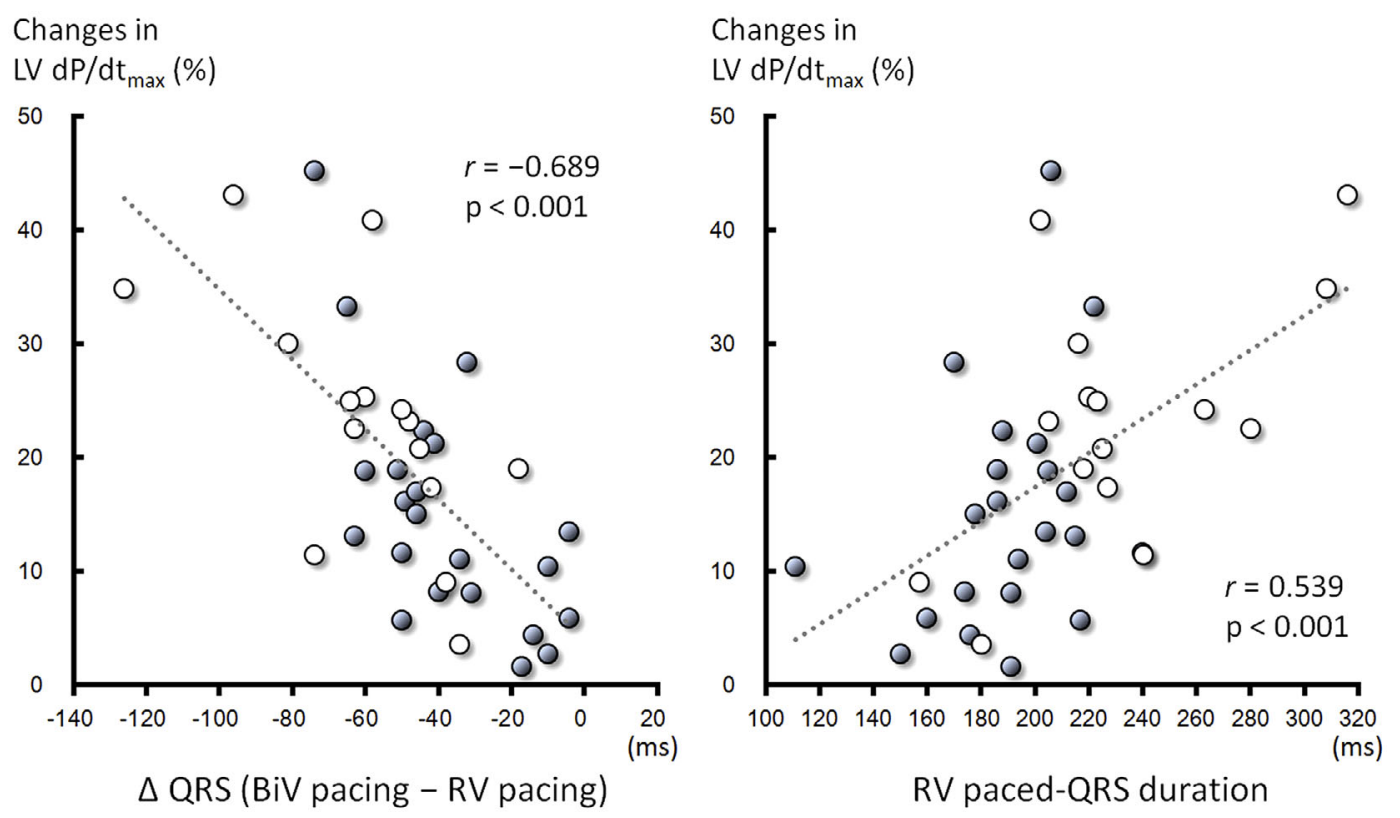

Figure 3. Relationship between left ventricular $\mathbf{d P / d t}$ max and QRS duration in switching from right ventricular apical pacing to biventricular pacing. The decrease in QRS duration affected by switching from right ventricular $(\mathrm{RV})$ apical pacing to biventricular $(\mathrm{BiV})$ pacing correlates with the increase

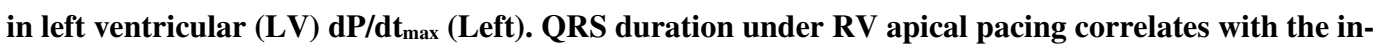

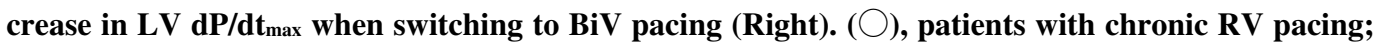
(O), patients with intrinsic conduction. Abbreviations: $\mathrm{dP}_{\mathbf{d}} \mathrm{dt}_{\max }$ : the maximum rate of $\mathrm{LV}$ pressure rise, tau: the time constant for isovolumic relaxation, $\mathrm{RV}$ : right ventricular, $\mathrm{LV}$ : left ventricular, $\mathrm{BiV}$ : biventricular

in the LV function are not expected. In contrast, BiV pacing is desirable in patients with systolic heart failure and a prolonged RV-paced QRS duration. In addition, our results are consistent with the opinion that BiV pacing should be considered in patients with heart failure who require RV pacing, if the QRS duration is prolonged by $\mathrm{RV}$ pacing.

\section{Study limitations}

First, the present study is limited by the small number of subjects and the retrospective nature of the study design. Second, mechanical dyssynchrony was not assessed because acute pacing tests were performed in our cardiac catheterization laboratory. Third, only short-term pacing data were analyzed in this study. Therefore, further studies are required to evaluate the long-term results. Fourth, the fixed AV delay setting may have influenced the measured QRS duration with a certain level of fusion beats.

\section{Conclusion}

Switching from RV apical pacing to BiV pacing improves the LV contractile function in proportion to the degree of QRS shortening, whereas the effect on LV diastolic relaxation varies. BiV pacing is recommended in patients with systolic heart failure and a prolonged RV-paced QRS duration. Furthermore, our results are consistent with the opinion that $\mathrm{BiV}$ pacing should be considered in patients with heart failure who require RV pacing, if the QRS duration is pro- longed by RV pacing.

The authors state that they have no Conflict of Interest (COI).

\section{Acknowledgment}

The authors thank Satoshi Nakatani, MD, Takashi Kurita, MD, Kazuhiro Suyama, MD, Naohiko Aihara, MD, Hideo Okamura, MD and Yuko Yamada, MD for their clinical expertise and cooperation. We also gratefully acknowledge the help and cooperation of the Electrophysiology Study Laboratory technicians, nurses and residents.

\section{References}

1. Kass DA, Chen $\mathrm{CH}$, Curry $\mathrm{C}$, et al. Improved left ventricular mechanics from acute VDD pacing in patients with dilated cardiomyopathy and ventricular conduction delay. Circulation 99: 15671573, 1999.

2. Wilkoff BL, Cook JR, Epstein AE, et al. Dual-chamber pacing or ventricular backup pacing in patients with an implantable defibrillator: the Dual Chamber and VVI Implantable Defibrillator (DAVID) Trial. JAMA 288: 3115-3123, 2002.

3. Shukla HH, Hellkamp AS, James EA, et al; Mode Selection Trial (MOST) Investigators. Heart failure hospitalization is more common in pacemaker patients with sinus node dysfunction and a prolonged paced QRS duration. Heart Rhythm 2: 245-251, 2005.

4. Varma N. Left ventricular conduction delays induced by right ventricular apical pacing: effect of left ventricular dysfunction and bundle branch block. J Cardiovasc Electrophysiol 19: 114-122, 2008.

5. Tops LF, Schalij MJ, Holman ER, et al. Right ventricular pacing 
can induce ventricular dyssynchrony in patients with atrial fibrillation after atrioventricular node ablation. J Am Coll Cardiol 48: 1642-1648, 2006.

6. Leon AR, Greenberg JM, Kanuru N, et al. Cardiac resynchronization in patients with congestive heart failure and chronic atrial fibrillation: effect of upgrading to biventricular pacing after chronic right ventricular pacing. J Am Coll Cardiol 39: 1258-1263, 2002.

7. Kindermann M, Hennen B, Jung J, et al. Biventricular versus conventional right ventricular stimulation for patients with standard pacing indication and left ventricular dysfunction: the Homburg Biventricular Pacing Evaluation (HOBIPACE). J Am Coll Cardiol 47: 1927-1937, 2006

8. Leclercq C, Cazeau S, Lellouche D, et al. Upgrading from single chamber right ventricular to biventricular pacing in permanently paced patients with worsening heart failure: The RD-CHF Study. Pacing Clin Electrophysiol 30 (Suppl 1): S23-S30, 2007.

9. Fröhlich G, Steffel J, Hürlimann D, et al. Upgrading to resynchronization therapy after chronic right ventricular pacing improves left ventricular remodelling. Eur Heart J 31: 1477-1485, 2010.

10. Auricchio A, Stellbrink C, Butter C, et al. Clinical efficacy of cardiac resynchronization therapy using left ventricular pacing in heart failure patients stratified by severity of ventricular conduction delay. J Am Coll Cardiol 42: 2109-2116, 2003.

11. Beshai JF, Grimm RA, Nagueh SF, et al. Cardiacresynchronization therapy in heart failure with narrow QRS complexes. N Engl J Med 357: 2461-2471, 2007.

12. Epstein AE, DiMarco JP, Ellenbogen KA, et al; American College of Cardiology Foundation; American Heart Association Task Force on Practice Guidelines; Heart Rhythm Society. 2012 ACCF/AHA/ HRS focused update incorporated into the ACCF/AHA/HRS 2008 guidelines for device-based therapy of cardiac rhythm abnormalities: a report of the American College of Cardiology Foundation/ American Heart Association Task Force on Practice Guidelines and the Heart Rhythm Society. Circulation 127: e283-e352, 2013.

13. McMurray JJ, Adamopoulos S, Anker SD, et al; ESC Committee for Practice Guidelines. ESC Guidelines for the diagnosis and treatment of acute and chronic heart failure 2012: The Task Force for the Diagnosis and Treatment of Acute and Chronic Heart Failure 2012 of the European Society of Cardiology. Developed in collaboration with the Heart Failure Association (HFA) of the ESC. Eur Heart J 33: 1787-1847, 2012.

14. Quinones MA, Gaasch WH, Alexander JK. Influence of acute changes in preload, afterload, contractile state and heart rate on ejection and isovolumic indices of myocardial contractility in man. Circulation 53: 293-302, 1976.

15. Frederiksen JW, Weiss JL, Weisfeldt ML. Time constant of isovolumic pressure fall: determinants in the working left ventricle. Am J Physiol 235: H701-H706, 1978.

16. Kashani A, Barold SS. Significance of QRS complex duration in patients with heart failure. J Am Coll Cardiol 46: 2183-2192, 2005.

17. Lecoq G, Leclercq C, Leray E, et al. Clinical and electrocardiographic predictors of a positive response to cardiac resynchronization therapy in advanced heart failure. Eur Heart J 26: 10941100, 2005.

18. Tamborero D, Vidal B, Tolosana JM, et al. Electrocardiographic versus echocardiographic optimization of the interventricular pacing delay in patients undergoing cardiac resynchronization therapy. J Cardiovasc Electrophysiol 22: 1129-1134, 2011.

19. Rickard J, Cheng A, Spragg D, et al. QRS narrowing is associated with reverse remodeling in patients with chronic right ventricular pacing upgraded to cardiac resynchronization therapy. Heart Rhythm 10: 55-60, 2013.

20. Donahue T, Niazi I, Leon A, Stucky M, Herrmann K; ESTEEMCRT Investigators. Acute and chronic response to CRT in narrow QRS patients. J Cardiovasc Transl Res 5: 232-241, 2012.

21. Ruschitzka F, Abraham WT, Singh JP, et al; EchoCRT Study Group. Cardiac-resynchronization therapy in heart failure with a narrow QRS complex. N Engl J Med 369: 1395-1405, 2013.

(C) 2015 The Japanese Society of Internal Medicine http://www.naika.or.jp/imonline/index.html 